JPN-ROK Joint Symposium in Veterinary Epidemiology

Session 1 "Highly pathogenic avian influenza (HPAI)"

\title{
Epidemiology of H5N8 Highly Pathogenic Avian Influenza (HPAl) in the Republic of Korea
}

\author{
Young-Myong Kang*, Wooseog Jeong, Sung-Chul Hwang, \\ Chung-Sik Jung Oun-Kyong Moon and Hong-Sik Park \\ Veterinary Epidemiology Division, Animal and Plant Quarantine Agency
}

On 16 January 2014, a suspected case of highly pathogenic avian influenza (HPAI) was reported in Gochang, Jeollabuk-do, located in south-western part of Korea. It had occurred sporadically some parts of Korea by 2015 and two cases were reported in 2016 . The country had previously experienced four major epidemics of HPAI in the years of 2003/2004, 2006/2007, 2008, and 2010/2011, and the type of all the isolated viruses was H5N1. From 2014 to 2016 epidemic, however, the virus type was identified as H5N8. During last 3 years, the outbreak of H5N8 had 3 waves (January 2014, September 2014, and September 2015). In this study, in order to understand epidemiological features of H5N8 HPAI virus, analysis were performed at three stages: country level, farms level and within farm level. Data were collected from the farm visiting sheet written by the animal controller of local veterinary service, and interviews with farmers. And we also considered spread patterns farm to farm and compared with previous H5N1 outbreaks. It was assumed that the virus was introduced into Korea through migratory birds which flied from high latitudes during the winter. At the initial phase of the outbreak, most farms, which were confirmed to be infected with HPAI H5N8 virus, were located in the vicinity of migratory bird habitats along the West Coast. It was supposed that the virus was transmitted between farms through the movement of farmers, visitors, vehicles and materials. HPAI H5N8 virus showed the differences of death patterns between chickens and ducks, very clearly. At the last phase of the outbreak, it occurred mainly on commercial ducks and Korean traditional broilers. It was assumed that the virus was introduced into Korea through migratory birds, and the major transmission routes between farms were movement of farmers, visitors, vehicles and materials. Although we were not easy to eradicate this virus because H5N8 virus has milder clinical signs and longer period of virus shedding, in comparison with previous H5N1 outbreaks, we are effort to eradicate this virus in many ways.

\footnotetext{
* Corresponding to: Young Myong Kang* 177 Hyeoksin 8-ro, Gimcheon, Gyeongsangbuk-do, 39660 Republic of Korea Tel: +82-54-912-0442; Fax: +82-54-912-0457

E-mail: kym34@korea.kr
} 